\title{
DISPERSION AND DAMPING OF SECOND SOUND IN NON-ISOTROPIC SOLIDS
}

\author{
PHILIP C. KWOK \\ IBM Watson Research Center, Yorktown Heights \\ New York \\ (Received 1 December 1966)
}

\begin{abstract}
The purpose of this note is to study the acoustic phonon collective mode or second sound mode in non-isotropic solids. Expressions for the velocity and damping of the second sound are given in terms of the acoustic phonon spectra and their relaxation times.
\end{abstract}

\section{Introduction}

IT IS well known $[1,2]$ that theoretically a slightly damped collective mode or second sound mode exists in an interacting phonon (acoustic) gas when the frequency $\omega$ satisfies $\omega \tau_{N} \ll 1$ and $\omega \tau_{U} \gg 1 . \tau_{N}$ is an average relaxation time describing the normal phonon collision processes that conserve momentum and $\tau_{U}$ is an average relaxation time describing all other momentum nonconserving processes, for example, Umklapp processes, boundary scattering and impurity scattering. Recently attempts have been made to observe this mode in solid Helium [3]. The velocity of second sound $u_{I I}$ in an isotropic system with only one type of phonon is $\left(u^{2 / 3}\right)^{1 / 2}$, where $u$ is the sound velocity. This was first calculated by Ward and Wilks [1] using the Boltzmann equation for the phonon distribution function and the conservation laws obtained from it. The calculation of $u_{I I}$ in an isotropic solid with three branches of acoustic phonons was carried out by Sussman and Thellung [4], it was found that

$$
u_{I I}=\left[\frac{1}{3} u_{l}^{2} u_{t}{ }^{2} \frac{u_{t}{ }^{3}+2 u_{l}{ }^{3}}{u_{t} 5+2 u_{l}^{5}}\right]^{1 / 2}
$$

where $u_{l}$ and $u_{t}$ are the longitudinal and transverse sound velocities. The microscopic consideration of second sound was treated by Kwok and Martin [2]. A general discussion of the connection between second sound, thermal conductivity and hydrodynamics was recently given by Guyer and Krumhansl [5]. In the present note we shall confine ourselves to the study of second sound from a practical point of view, deriving the dispersion and damping of this collective mode in solids with anisotropic phonon spectra [6]. We shall simplify our calculations by neglecting all phonon momentum-destroying processes, i.e. letting $\tau_{U} \rightarrow \infty$ In Section 2, the Boltzman equation and the consequent conservation laws which will be used for the derivation are discussed. In Section 3, the dispersion relation of the second sound is derived. In Section 4, the damping of this collective mode is considered. 


\section{Boltzmann Equation}

We shall begin by examining the Boltzmann equation for the phonon distribution functions. microscopic justification of the use of this equation may be found in reference 2. The Boltzmann equation is

$$
\frac{\partial}{\partial t} N_{\mathbf{k} s}+\mathbf{u}_{\mathrm{k} s} \cdot \nabla N_{\mathrm{k} s}=-\left(\frac{\partial N_{\mathrm{k} s}}{\partial_{t}}\right)_{\mathrm{co11}}
$$

where $N_{\mathbf{k} s}$ is the distribution for the phonon with momentum $\mathbf{k}(\hbar=1)$ and polarization $5 ; \mathbf{k}_{\mathbf{k}} s$ is the group velocity defined from the frequency $\omega_{\mathbf{k}} s$ according to

$$
\mathbf{u}_{\mathbf{k} s}=\nabla_{\mathbf{k}} \omega_{\mathbf{k}} s
$$

The collision term in (1) contains all normal collision processes, and has the following form when only the normal three-phonon processes are taken into account [7]

$$
\begin{aligned}
\left(\frac{\partial N_{\mathbf{k} s}}{\partial t}\right)_{c \circ 11 .}=-\frac{\pi}{4 \rho} & \sum 2 \mid A_{\mathbf{k} s, \mathbf{k}^{\prime} s^{\prime},-\left.\mathbf{k}^{\prime \prime} s^{\prime \prime}\right|^{2} \omega \omega^{\prime} \omega^{\prime \prime} \mathrm{x}} \\
& \times \delta\left(\omega+\omega^{\prime}-\omega^{\prime \prime}\right)\left[(N+1)\left(N^{\prime}+1\right) N^{\prime \prime}-\right. \\
& \left.-N N^{\prime}\left(N^{\prime \prime}+1\right)\right]- \\
& -\frac{\pi}{4 \rho} \quad \sum \mid A_{-\mathbf{k} s, \mathbf{k}^{\prime} s^{\prime},\left.\mathbf{k}^{\prime \prime} s^{\prime \prime}\right|^{2} \omega^{\prime} \omega^{\prime} \omega^{\prime \prime} \mathrm{x}} \\
& \times \delta\left(\omega-\omega^{\prime}-\omega^{\prime \prime}\right)\left[(N+1) N^{\prime} N^{\prime \prime}-\right. \\
= & \left.N\left(N^{\prime}+1\right)\left(N^{\prime \prime}+1\right)\right]
\end{aligned}
$$

where we have used the abbreviation $\omega^{\prime}=\omega_{k} s^{\prime}, N^{\prime}=N_{k} s^{\prime}$, etc. $\rho$ is the mass density of the solid and $A_{\mathbf{k}_{1}} s_{1}, \mathbf{k}_{2} s_{2}, \mathbf{k}_{3} s_{3}$ is the cubic anharmonic coupling parameter which is zero unless $\mathbf{k}_{1}+\mathbf{k}_{2}+\mathbf{k}_{3}=\mathbf{0}$ since we are only considering normal processes. Because the normal processes conserve both the energy and momentum of the participating phonons, we can deduce from (1) the following conservation laws (in particular this can be directly verified for the approximate collision term given in (3))

$$
\begin{array}{r}
\frac{\partial}{\partial t} E(\mathbf{r} t)+\nabla_{\alpha j_{\alpha}}^{E}(\mathbf{r} t)=0 \\
\frac{\partial}{\partial t} P_{\alpha}(\mathbf{r} t)+\nabla_{\beta} T_{\alpha \beta}(\mathbf{r} t)=0
\end{array}
$$


where $E(\mathbf{r} t)$ and $P_{\alpha}(\mathbf{r} t)$ are the energy and momentum density of the phonons

$$
\begin{aligned}
& E(\mathbf{r} t)=\sum_{\mathbf{k} s} \omega_{\mathbf{k} s} N_{\mathbf{k} s} \\
& P_{\alpha}(\mathbf{r} t)=\sum_{\mathbf{k} s} k_{\alpha} N_{\mathbf{k} s}
\end{aligned}
$$

and $j_{\alpha}^{E}(\mathbf{r} t)$ and $T_{\alpha \beta}(\mathbf{r} t)$ are the energy current and momentum flux tensor

$$
\begin{aligned}
& j_{\alpha}^{E}(\mathbf{r} t)=\sum_{\mathbf{k} s} \omega_{\mathbf{k} s} u_{\mathbf{k} s}^{\alpha} N_{\mathbf{k} s} \\
& T_{\alpha \beta}(\mathbf{r} t)=\sum_{\mathbf{k} s} k_{\alpha} u_{\mathbf{k} s}^{\beta} N_{\mathbf{k} s} .
\end{aligned}
$$

\section{Dispersion Relation of Second Sound}

The dispersion of the collective mode that is implied by the conservation laws (4) and (5) will now be calculated; the damping of this mode will be considered in the next section. Following usual procedure [2] we first find the quasi- or local-equilibrium phonon distribution functions $\tilde{N}_{\mathbf{k} s}$. These functions are defined as the functions that make the collision term (3) vanish identically. It is easily shown that $\tilde{N}_{\mathbf{k} s}$ have the following form

$$
\tilde{N}_{\mathbf{k} s}=\left[\exp \left(\frac{\omega_{\mathbf{k} s}}{k_{B} T(\mathbf{r} t)}-\frac{\mathbf{k} \cdot \mathbf{V}(\mathbf{r} t)}{k_{B} T_{0}}\right)-1\right]^{-1}
$$

where $T(\mathbf{r} t)$ and $\mathbf{V}(\mathbf{r} t)$ can be arbitrary functions of $\mathbf{r}, t . T_{0}$ is chosen to be the ambient temperature or thermal equilibrium temperature of the phonon system. $T(\mathbf{r} t)$ and $\mathbf{V}(\mathbf{r} t)$ may be interpreted as the local temperature and local drift velocity of the phonons if

$\left|T(\mathbf{r} t)-T_{0}\right| \ll T_{0}$ and $|\mathbf{V}(\mathbf{r} t)| \ll$ the sound velocities. We shall assume that the deviation from complete thermal equilibrium is small (collision-dominated situation). Then $\tilde{N}_{\mathbf{k} s}$ can be expanded and we obtain

$$
\tilde{N}_{\mathbf{k} s}=N_{\mathbf{k} s}^{\circ}+N_{\mathbf{k} s}^{\circ}\left(N_{\mathbf{k} s}^{\circ}+1\right) \frac{1}{k_{B} T_{0}}\left(\omega_{\mathbf{k} s} \sigma(\mathbf{r} t)+\mathbf{k} \cdot \mathbf{V}(\mathbf{r} t)\right)
$$

to linear order in $\sigma(\mathbf{r} t)=\frac{T(r t)-T_{0}}{T_{0}}$ and $\mathbf{V}(\mathbf{r} t)$, where $\widetilde{N}_{\mathbf{k} s}^{o}$ is the thermal equilibrium phonon distribution function at temperature $T_{0},\left[\exp \left(\frac{\omega_{k} s}{k_{B} T_{0}}\right)-1\right]^{-1} \cdot$ These functions $\sigma(\mathbf{r} t)$ and $\mathbf{V}(\mathbf{r} t)$ are not independent but are related to each other through the conservation laws (4) and (5). In fact the second sound made is deduced from the linearized 
equations that couple these functions. To obtain the undamped second sound mode one approximates the thermodynamic quantities in (4) and (5) by their quasi-equilibrium values which are given by (6)-(9) with $N_{k s}$ replaced by $N_{k s}$. They can then be expressed in terms of $\sigma$ and $V$. Te find

$$
\begin{aligned}
& \widetilde{E}(\mathbf{r} t)=E^{0}+A \sigma(\mathbf{r} t) \\
& \tilde{j}_{\alpha}^{E}(\mathbf{r} t)=B_{\alpha \beta} V_{\beta}(\mathbf{r} t) \\
& \tilde{P}_{\alpha}(\mathbf{r} t)=C_{\alpha \beta} V_{\beta}(\mathbf{r} t) \\
& \tilde{T}_{\alpha \beta}(\mathbf{r} t)=T_{\alpha \beta}^{0}+B_{\beta \alpha} \sigma(\mathbf{r} t)
\end{aligned}
$$

where $E^{\circ}$ and $T_{\alpha \beta}^{\circ}$ are the thermal equilibrium values of $E$ and $T_{\alpha \beta}$, and we have used the fact that $\omega_{k s}=\omega_{-k s}$. The temperature-dependent coefficients $A, B$ and $C$ are

$$
\begin{aligned}
& A=\frac{1}{k_{B} T_{0}} \sum_{\mathbf{k} s} S_{\mathbf{k} s}^{0} \stackrel{2}{\omega_{\mathbf{k} s}} \\
& B_{\alpha \beta}=\frac{1}{k_{B} T_{0}} \sum_{\mathbf{k} s} S_{\mathbf{k} s}^{0} \underset{u_{\mathrm{k} s} k_{\beta}}{\dot{k}} k \\
& C_{\alpha \beta}=\frac{1}{k_{B} T_{0}} \sum_{\mathbf{k} s} S_{\mathbf{k} s}^{0} k_{\alpha} k_{\beta}
\end{aligned}
$$

with

$$
S_{\mathbf{k} s}^{0}=N_{\mathbf{k} s}^{0}\left(N_{\mathbf{k} s}^{0}+1\right) \text {. }
$$

The equations satisfied by $\sigma$ and $\mathbf{V}$ are then readily obtained to be

$$
\begin{aligned}
& A \frac{\partial}{\partial t} \sigma(\mathbf{r} t)+B_{\alpha \beta} \nabla_{\alpha} V_{\beta}(\mathbf{r} t)=0 \\
& C_{\alpha \beta} \frac{\partial}{\partial t} V_{\beta}(\mathbf{r} t)+B_{\beta \alpha} \nabla_{\beta} \sigma(\mathbf{r} t)=0 .
\end{aligned}
$$

These equations are considerably simplified for a solid with cubic symmetry because the matrices $B$ and $C$ are diagonal.

$$
B_{\alpha \beta}=\delta_{\alpha \beta} B ; \quad C_{\alpha \beta}=\delta_{\alpha \beta} C \quad \text { (cubic) }
$$




$$
\begin{aligned}
& B=\frac{1}{3 k_{B} T_{0}} \sum_{\mathbf{k} s} S_{\mathbf{k} s}^{\circ}\left(\mathbf{k} \cdot \mathbf{u}_{\mathbf{k} s}\right) \\
& C=\frac{1}{3 k_{B} T_{0}} \sum_{\mathbf{k} s} S_{\mathbf{k} s}^{\circ}{ }^{2} .
\end{aligned}
$$

In general these matrices are not diagonal. To carry on the derivation we shall make the reasonable assumption that $C_{0 \beta}$ has an inverse. Then $(20)$ and (21) can be rewritten as

$$
\begin{aligned}
& \frac{\partial}{\partial t} \sigma(\mathbf{r} t)+\frac{B_{\alpha \beta}}{A} \nabla_{\beta} V_{\beta}(\mathbf{r} t)=0 \\
& \frac{\partial}{\partial t} V_{\alpha}(\mathbf{r} t)+C_{\alpha \beta}^{-1} B_{\gamma \beta} \nabla_{\gamma} \sigma(\mathbf{r} t)=0 .
\end{aligned}
$$

We shall look for wave solutions of the form

$$
\begin{aligned}
& \sigma(\mathbf{r} t)=\sigma e^{i p \cdot r-i \omega t} \\
& V_{\alpha}(\mathbf{r} t)=V_{\alpha} e^{i p \cdot r i \omega t}
\end{aligned}
$$

Substituting (25) into $\left(20^{\circ}\right)$ and $\left(21^{\prime}\right)$ we obtain

$$
\left[\omega^{2}-p^{2}\left(\frac{1}{A} U_{\alpha}(\hat{p}) C_{\alpha \beta}^{-1} U_{\beta}(\hat{p})\right)\right] \sigma=0
$$

and

$$
\left[\omega^{2} \delta_{\alpha \beta}-p^{2} \frac{1}{A}\left(C_{\alpha \gamma}^{-1} U_{\gamma}(\hat{p})\right) U_{\beta}(\hat{p})\right] V_{\beta}=0
$$

with

$$
\sigma=\frac{p}{\omega} \frac{U_{\alpha}}{A} V_{\alpha} ; \quad \hat{p}=\frac{\mathbf{P}}{P}
$$

where $p$ is the magnitude of $\mathbf{P}$ and $U_{\beta}(\hat{p})$ is a vector dependent on the direction of $\hat{\mathbf{p}}$ given by

$$
U_{\beta}(\hat{p})=B_{\gamma \beta} \hat{p}_{\gamma}
$$

Equation (26) describes the propagation of temperature variation or phonon energy density variation and equation (27) describes the propagation of phonon momentum density. From (26) we 
deduce immediately the dispersion of the heat conduction made or second sound mode. We find

$$
\begin{aligned}
& \omega^{2}=u_{I I}^{2}(\hat{p}) p^{2} \\
& u_{I I}^{2}(\hat{p})=\frac{1}{A} U_{\alpha} C_{\alpha \beta}^{-1} U_{\beta} .
\end{aligned}
$$

From (27) we observe that there are three eigenmodes. Two eigenmodes correspond to static solutions with $\omega^{2}=0$ and eigenvectors along any one of the orthogonal directions $\hat{e}^{1}$ and $\hat{e}^{2}$ perpendicular to $\mathbf{U}$ or $\hat{U}$. These modes do not involve heat propagation since according to (28) $\sigma$ wil vanish. The third eigenmode which is associated with heat propagation has the same dispersion as (30) and the eigenvector is

$$
\mathbf{V}^{(3)}=V_{1} \hat{\mathbf{e}}^{1}+V_{2} \hat{\mathbf{e}}^{2}+V_{3} \hat{U}
$$

where $V_{3}$ is an arbitrary constant, and $V_{1}$ and $V_{2}$ are given by

$$
V_{1}=\frac{e_{\alpha}^{1}\left(C_{\alpha \gamma}^{-1} U_{\gamma}\right)}{U_{\alpha}\left(C_{\alpha \gamma}^{-1} U_{\gamma}\right)} V_{3} ; \quad V_{2}=\frac{e_{\alpha}^{2}\left(C_{\alpha \gamma}^{-1} U_{\gamma}\right)}{U_{\alpha}\left(C_{\alpha \gamma}^{-1} U_{\gamma}\right)} V_{3} .
$$

The eigenvectors are not orthogonal because the matrix $\frac{1}{A} C_{\alpha \gamma}^{-1} U_{\gamma} U_{\beta}$ in (27) is not hermitian (symmetric). Our general conclusion is, therefore, that heat propagating along a $\hat{p}$ direction has a velocity $u_{1 I}$ that is a function of $\hat{p}$ and that the phonon momentum density associated with this mode is pointed along $C_{\alpha \beta} \mathbf{V}_{\beta}{ }^{\left({ }^{3}\right)}$, whose direction is in general different from $\hat{p}$. For a cubic crystal however $C_{\alpha \beta} V_{\beta}{ }^{(3)}$ is along $\hat{p}$ and $u_{\text {II }}$ is independent of $\hat{p}$

$$
\begin{aligned}
u_{\mathrm{II}}^{2} & =\frac{B^{2}}{A C} \\
& =\frac{1}{3}\left(\sum_{\mathbf{k} s} S_{\mathbf{k} s}^{0}\left(\mathbf{k} \cdot \mathbf{u}_{\mathbf{k} s}\right)\right)^{2} /\left(\sum_{\mathbf{k} s} S_{\mathbf{k} s}^{0} \omega_{\mathbf{k} s}^{2}\right)\left(\sum_{\mathbf{k} s} S_{\mathbf{k} s}^{\circ} k^{2}\right) .
\end{aligned}
$$

Furthermore, if we neglect the dispersion of the phonon spectra, i.e. using $\omega_{\mathbf{k}} \simeq \simeq \mathbf{k}$. $\left(\begin{array}{ll}\nabla_{\mathbf{k}} & \omega_{\mathbf{k}} s \\ k \rightarrow 0\end{array}\right) \mathbf{k} \cdot(\underset{k}{\mathbf{k} s})_{k \rightarrow 0}$ we obtain

$$
\begin{array}{rlrl}
u_{\mathrm{II}}^{2} & =\frac{1}{3}\left(\sum_{\mathbf{k} s} S_{\mathbf{k} s}^{\circ} \omega_{\mathbf{k} s}^{2} / \sum_{\mathbf{k} s} S_{\mathbf{k} s}^{\circ} k^{2}\right) \\
& =\frac{1}{3} \overline{u^{2}} \quad & \text { (cubic). }
\end{array}
$$


For other crystals $V^{(3)}$ may still be proportional to $\hat{p}$ if $\hat{p}$ is along high symmetry directions but $u_{I I}$ will in general be different

\section{Damping of Second Sound}

We will now consider the damping of the second sound mode whose dispersion we have calculated in the previous section. To obtain the damping one has to go beyond the quasi-equilibriun approximation. One has to calculate the deviation of the thermodynamic functions $E, \mathrm{j}^{E}$ etc. from their quasi-equilibrium values or the deviation of $N_{\mathbf{k} s}$ from $\tilde{N}_{\mathbf{k s}}$. Following the usual procedure we write

$$
N_{\mathbf{k} s}=\tilde{N}_{\mathbf{k} s}+n_{\mathbf{k} s}
$$

and substitute it into the Boltzmann equation (1). Linearizing and using the relaxation time approximation [8], i.e. replacing $-\left(\frac{\partial N_{k} s}{\partial t}\right)_{c \circ 11}$ by $-\frac{n_{k} s}{\tau_{k s}}$ where $\tau_{k s}$ is the lifetime of the phonon $(\mathbf{k}, s)$ we obtain

$$
n_{\mathbf{k} s}=-\tau_{\mathbf{k} s}\left[\frac{\partial}{\partial t} \tilde{N}_{\mathbf{k} s}+u_{\mathbf{k} s} \cdot \nabla \tilde{N}_{\mathbf{k} s}\right]
$$

(37) can be put in a more convenient form if we make use of the expansion (11) and equations $\left(20^{\prime}\right)$ and $\left(21^{\prime}\right)$. The result is

$$
\begin{aligned}
n_{\mathbf{k} s}=-\tau_{\mathbf{k} s} \frac{S_{\mathbf{k} s}^{\circ}}{k_{B} T_{0}} & {\left[\left(u_{\mathbf{k} s}^{\alpha} k_{\beta}-\frac{\omega_{\mathbf{k} s} B_{\alpha \beta}}{A}\right) \nabla_{\alpha} V_{\beta}\right.} \\
& \left.+\left(\omega_{\mathbf{k} s} u_{\mathbf{k} s}^{\alpha}-k_{\beta} C_{\beta \gamma}^{-1} B_{\alpha \gamma}\right) \nabla_{\alpha} \sigma\right]
\end{aligned}
$$

From this expression one can calculate the deviation of the quantities $E, j_{\alpha}^{E}$ etc. from their quasi-equilibrium values in terms of $\sigma$ and $\mathbf{V}$ (and the relaxation times $\tau_{k} s$ ):

$$
\begin{aligned}
& E(\mathbf{r} t)=\widetilde{E}(\mathbf{r} t)+\delta E(\mathbf{r} t) \\
& \delta E(\mathbf{r} t)=\sum_{\mathbf{k} s} \omega_{\mathbf{k} s} n_{\mathbf{k} s}
\end{aligned}
$$

The dispersion of the second sound with damping may now be computed upon using (4) and (5) again. The expressions for $\delta E$ etc. in terms of $\sigma$ and $\mathbf{V}$ in general involve very complicated tensorial quantities which cannot be simplified. For illustrative purposes we shall confine 
ourselves to the simplest case of a cubic crystal. Then we find, neglecting phonon dispersion,

$$
\begin{aligned}
& \delta E(\mathbf{r} t)=0 \\
& \delta j_{\alpha}^{E}(\mathbf{r} t)=-\bar{u}^{2} \kappa_{J} \nabla_{\alpha} \sigma(\mathbf{r} t) \\
& \delta P_{\alpha}(\mathbf{r} t)=-\kappa_{P} \nabla_{\alpha} \sigma(\mathbf{r} t) \\
& \delta T_{\alpha \beta}(\mathbf{r} t)=\frac{2}{3} \eta \delta_{\alpha \beta}(\nabla \cdot \mathbf{V}(\mathbf{r} t))-\eta\left(\nabla_{\alpha} V_{\beta}+\nabla_{\beta} V_{\alpha}\right)
\end{aligned}
$$

where $\overline{u^{2}}$ is defined in (35) and the transport coefficients $k_{J}, k_{P}$ and $\eta$ are

$$
\begin{aligned}
& k_{J}=\frac{1}{3 u^{2} k_{B} T_{0}} \sum_{\mathbf{k} s} S_{\mathbf{k} s}^{0} \omega_{\mathbf{k} s}\left(u_{\mathbf{k} s}^{2}-\overline{u^{2}}\right) \tau_{\mathbf{k} s} \\
& k_{P}=\frac{1}{3 k_{B} T_{0}} \sum_{\mathbf{k} s} S_{\mathbf{k} s k^{2}\left(u_{\mathbf{k} s}^{2}-\overline{u^{2}}\right) \tau_{\mathbf{k} s}}^{0} \\
& \eta=\frac{1}{15 k_{B} T_{0}} \sum_{\mathbf{k} s} S_{\mathbf{k} s \omega_{\mathbf{k} s}^{2} \tau_{\mathbf{k} s}}^{0}
\end{aligned}
$$

The conservation laws (4) and (5) become

$$
\begin{gathered}
A \frac{\partial}{\partial t} \sigma(\mathbf{r} t)+B \nabla \cdot \mathbf{V}(\mathbf{r} t)-\bar{u}^{2} \kappa_{J} \nabla^{2} \sigma(\mathbf{r} t)=0 \\
C \frac{\partial}{\partial t} V_{\alpha}(\mathbf{r} t)-\kappa_{P} \nabla_{\alpha} \frac{\partial}{\partial t} \sigma(\mathbf{r} t)+B \nabla_{\alpha} \sigma(\mathbf{r} t) \\
-\frac{\eta}{3} \nabla_{\alpha}\left(\nabla \cdot \mathbf{V}(\mathbf{r} t)-\eta \nabla^{2} V_{\alpha}(\mathbf{r} t)=0 .\right.
\end{gathered}
$$

The dispersion relation of the second mode can now be readily obtained to be

where

$$
\omega^{2}+i \omega D_{\mathrm{II}} k^{2}-u_{\mathrm{II}}^{2} k^{2}=0
$$

$$
D_{\mathrm{II}}=\frac{u_{\mathrm{II}}^{2}}{A}\left[4 \eta+\left(K_{J}-K_{P}\right)\right]
$$


The damping of the second sound is therefore

$$
\gamma_{\mathrm{II}}=\frac{\omega L_{I I \mathrm{I}}{ }^{2}}{2 u_{\mathrm{II}} \mathrm{I}^{k}}=\omega^{2} \frac{1}{A}\left[6 \eta+\frac{3}{2}\left(\kappa_{J}-\kappa_{P}\right)\right]
$$

proportional to the square of the frequency. To determine its temperature dependence one has to know the dependence of $\tau_{k} s$ on the magnitude of $k$. Assuming it is given by three-phonon processes then $\tau_{k s}$ is proportional to $\frac{1}{T_{0}} r_{k} 5-r$ where $r$ is an integer $0 \leqslant r \leqslant 4$ [9]. At low temperatures $T_{0} \ll$ Debye temperature, the transport coefficients $\mathrm{KJ}_{\mathrm{J}}, \mathrm{KP}_{\mathrm{P}}$ and $\eta$ are inversely proportional to $T_{0}$ for any $r$; and $A$ is proportional to $T_{0}{ }^{4}$. Therefore $\gamma_{I I}$ is proportional to $T_{0}{ }^{-5}$.

\section{Acknowledgement}

I would like to thank Dr. P.B. Miller for a helpful discussion on the subject.

\section{References}

1. J.C. WARD and J. MILKS, Phil. Mag. 43, 48 (1952).

2. P.C. KWOK and P.C. MARTIN, Phys, Rev. 142, 495 (1966).

3. C.C. ACKerman, B. Bertman, H. A. Fairbank and R.A. GUYer, Phys. Rev. Letters 16, 789 (1966).

4. J.A. SUSSman and A. Thellung, Proc. Phys. Soc. 81, 1122 (1963).

5. R.A. GUYER and J.A. KRUMHANSL (2 papers to be published in Phys. Rev.).

6. Similar work using a slightly different approach has been carried out independently by . R.N. GURZHI, Fizika tverd. Tela 7, 3515 (1965) (Trans: Soviet Phys. solid St. u, 2838 (1966)).

7. See for example R.E. PEIERLS, Quantum Theory of Solids. Pergamon Press, Oxford (1955).

8. In the case of a single phonon branch with an isotropic and dispersionless spectrum the relaxation time approximation can be easily shown to be equivalent to the more fundamental approach of Guyer and Krumhansl (Ref. 5) in calculating the damping of second sound. It is the author's opinion that such close connection still exists in the general case under consideration.

9. See for example C. HERring, Phys. Rev. 95, 954 (1954). 